\title{
Daily nursing care on patients undergoing venous-venous extracorporeal membrane oxygenation: a challenging procedure!
}

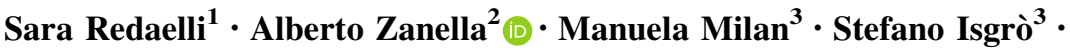 \\ Alberto Lucchini ${ }^{3} \cdot$ Antonio Pesenti $^{2,4} \cdot$ Nicolò Patroniti $^{3,5}$
}

Received: 15 October 2015/Accepted: 6 June 2016/Published online: 16 June 2016

(C) The Japanese Society for Artificial Organs 2016

\begin{abstract}
Daily nursing in critical care patients may alter vital parameters, especially in the most critically ill patients. The aim of our study was to evaluate feasibility and safety of daily nursing on patients undergoing venousvenous extracorporeal membrane oxygenation (vv-ECMO) for severe respiratory failure. Daily nursing was performed following defined phases (sponge bath, elevation with scooping stretcher, change position of endotracheal tube, dressing replacement). We recorded physiological and ECMO parameters before and during daily nursing in 5 patients for several days (total: 25 daily nursing) and adverse events: desaturation, hypertension, reduction of mixed venous oxygen saturation, arterial oxygen saturation or ECMO blood flow and elevation in minute ventilation. Sedative drug dosage and additional bolus were recorded. Daily nursing was performed in $92 \%$ of cases $(23 / 25)$,
\end{abstract}

Electronic supplementary material The online version of this article (doi:10.1007/s10047-016-0912-y) contains supplementary material, which is available to authorized users.

Alberto Zanella

zanella.alb@gmail.com

1 U.O. di anestesia e terapia intensiva, Presidio ospedaliero S. Chiara, Ospedale di Trento, Trento, Italy

2 Dipartimento di Anestesia, Rianimazione ed Emergenza Urgenza, Fondazione IRCCS Ca' Granda-Ospedale Maggiore Policlinico, Via Francesco Sforza 35, 20122 Milan, MI, Italy

3 Dipartimento di Emergenza e Urgenza, Ospedale San Gerardo, Monza, Italy

4 Dipartimento di Fisiopatologia medico-chirurgica e dei trapianti, Università degli Studi di Milano, Via Francesco Sforza 35, Milan, Italy

5 Dipartimento di Medicina e Chirurgia, Università degli Studi di Milano Bicocca, Monza, MB, Italy with a minimum of two adverse events per daily nursing. Hypertension and tachycardia were mostly recorded at the beginning, while desaturation, reduction in mixed venous oxygen saturation and blood flow were recorded during elevation with scooping stretcher. Increase in minute ventilation was frequent in spontaneous breathing patients. Additional bolus of sedation was required before and/or during nursing. Daily nursing significantly alters physiologic parameters; thus, it should be performed only when physicians are readily available to treat adverse events.

Keywords ECMO - Daily nursing care · ARDS

\section{Introduction}

Daily nursing of critical patients admitted to the intensive care unit (ICU) is a fundamental intervention to improve patient hygiene, ameliorate patient comfort, prevent iatrogenic infections and assess skin integrity $[1,2]$. Few data are available on daily nursing care in ICU; recently, Coyer et al. described an Australian ICU bed bath practice consisting of a sponge bath with soap and water, sheet replacement and position change. Other reports showed a temporarily increase in "work of breathing" and oxygen consumption during daily nursing care activities (bed baths and turning-repositioning) in critically ill patients [2-11]. More recently, Happ et al. raised several concerns about performing bed bath in patients undergoing weaning trial, underlining the importance of collaboration between nurses and clinicians to define which patients could tolerate nursing care and when to perform it [12]. No studies addressed daily nursing care in patients with severe acute respiratory distress syndrome (ARDS) during veno-venous extracorporeal membrane oxygenation (ECMO) support. 
These patients are generally unstable and nursing care may be even more challenging and hazardous due to precarious oxygenation, anticoagulation, unstable extracorporeal blood flow and possible incidental decannulation.

Our center has a long tradition of veno-venous ECMO (vv-ECMO) support and, in the last few years, we have further increased the number of patients connected to ECMO by-pass due to the development of the Italian ECMO Network (ECMOnet) following the H1N1 pandemic in 2009 [13-15]. Over time we have developed a protocol for daily nursing care in severely hypoxic patients connected to vv-ECMO.

The aim of our study is to evaluate the feasibility and safety of daily nursing care on patients undergoing vvECMO for severe respiratory failure.

\section{Materials and methods}

We conducted a prospective observational study between November 2009 and January 2010 in our ten-beds general ICU at the San Gerardo Hospital, Monza, Italy. The study was approved by the ethical committee of our institution. Our ICU has been performing vv-ECMO in ARDS patients for more than twenty years during which we have developed and refined a specific daily nursing protocol for the most severe critically ill patients. Nursing can be performed only if sedation level is adequate and hemodynamic and extracorporeal blood flow (BF) are stable while arterial saturation has to be above $85 \%$. The presence of physicians or perfusionists during nursing care is not required, but an intensivist has to be readily available. Boluses of sedation can be administered before or during nursing only by physicians. The daily nursing protocol consists of four phases in fixed order: (1) sponge bath, (2) elevation with scooping stretcher for sheets replacement and back hygiene, (3) change position of endotracheal tube (ETT) and (4) dressing replacement that needs to be performed at least every 7 days or whenever necessary. Since mobilization of the patient during daily nursing has to be performed by 2 or 3 nurses and at least one nurse has to be a senior nurse, the order of the phases can be changed, according to ICU emergencies and commitments. Sponge bath consisted of a complete bed bath with disposable presoaped sponges and warm water $\left(37^{\circ} \mathrm{C}\right)$, shave, eyes care and oral hygiene performed with a special suction cannula incorporating a toothbrush and chlorhexidine $0.2 \%$ mouthwash. If clyster is required, bedpan is employed. Back hygiene and sheets replacement are performed by lifting the patients over the bed with a scooping stretcher attached to a lift so as to minimize the mobilization of patients and side turning, which could affect the blood drainage, thus reducing the blood flow. During elevation with scooping stretcher, two nurses performed the maneuver while a third one was responsible for the ECMO circuit to avoid tractions on cannulas or kinking of the circuit.

All cannulas dressing (high adhesiveness transparent semi-permeable dressing, Tegaderm Advanced-3M Healthcare) is renewed at least every 7 days or earlier if not adherent or in case of bleeding. Cannulation sites are disinfected with a skin disinfectant, $2 \%$ chlorhexidine gluconate in $70 \%$ isopropyl alcohol (Nex Medical-Italy). All cannulas are secured to the skin by two sutureless grips (GripLock-Vygon). Prevention of pressure sores due to contact of the circuit with the patient's skin was achieved by applying hydrocolloid ultrathin film (Duoderm extrathin-Convatec) on the skin.

In the present study, we observed five patients undergoing vv-ECMO over several days during the daily nursing care for a total of 25 daily nursing episodes. All the patients were connected to a PLS System (Maquet), consisting of a polymethylpentene oxygenator, a centrifugal pump, and a heater unit. All the pumps were set to deliver a constant number of rpm; thus, blood flow could change with resistances. Several physiological parameters were continuously recorded [heart rate (HR), systolic blood pressure (SBP), mixed venous saturation $\left(\mathrm{SvO}_{2}\right)$, arterial oxygen saturation $\left(\mathrm{SpO}_{2}\right)$, tidal volume (TV), respiratory rate (RR), minute ventilation (MV) and positive end-expiratory pressure (PEEP)] into the ICU electronic chart (Dräger Innovian, VF5.4 2006 Dräger Medical systems). We manually collected changes in ECMO blood flow (BF) during each step of the nursing protocol. Level of sedation was assessed before nursing with bispectral index (BIS) in paralyzed patients, otherwise with Ramsay sedation scale (RSS); sedative drug dosage and additional bolus of sedation before or during nursing were noted. All data were recorded from 15 min before the start of nursing until the end of the procedure; parameters were extracted from ICU electronic chart every minute; each minute value was the last recorded over the minute.

Five alterations from baseline of vital signs, lasting at least $2 \mathrm{~min}$, were considered as adverse events as follows. Baseline was defined as the mean value of each physiological parameter recorded over the $3 \mathrm{~min}$ before the beginning of the adverse event. Adverse events were:

- Desaturation: reduction in $\mathrm{SpO}_{2}$ equal to or greater than 3 points percentage.

- Hypertension: elevation in systolic blood pressure greater than $15 \%$ from baseline.

- $\mathrm{SvO}_{2}$ reduction: reduction in $\mathrm{SvO}_{2}$ greater than 3 points percentage from baseline.

- Tachycardia: elevation in heart rate greater than $15 \%$ from baseline. 
- $\mathrm{BF}$ reduction: reduction in $\mathrm{BF}$ greater than $15 \%$ from baseline.

In assisted mechanical ventilation patients, we also recorded alteration of respiratory pattern as follows:

- Tachypnea: elevation in respiratory rate greater than $30 \%$ from baseline.

- TV reduction: reduction in TV greater than $25 \%$ from baseline.

- TV elevation: elevation in TV greater than $25 \%$ from baseline.

- MV elevation: elevation in MV greater than $25 \%$ from baseline.

Each adverse event was considered overcome when the parameter returned to baseline values.

\section{Statistics}

All data are expressed as mean \pm standard deviation or median (25-75 percentile).

Variations in vital parameters from baseline were performed with paired $t$ test (for positive Shapiro-Wilk normality test) or with Wilcoxon signed rank test (for negative Shapiro-Wilk normality test). Correlations were performed with the Pearson product moment correlation.

\section{Results}

Patients characteristics are summarized in Table 1, while baseline conditions before nursing episodes are summarized in Table 2. All the patients suffered from severe hypoxemic conditions due to ARDS H1N1 related, 3 were supported with femoro-femoral vv-ECMO and 2 with femoro-jugular vv-ECMO. Average BF and RPM were $3.1 \pm 1$ and $2532 \pm 464 \mathrm{~L} / \mathrm{min}$, respectively. Daily

Table 1 Demographic data

\begin{tabular}{ll}
\hline Diagnosis & ARDS H1N1 related \\
\hline Age (years) & $39 \pm 14$ \\
Weight $(\mathrm{kg})$ & $89 \pm 27$ \\
Male & $3 / 5$ \\
SAPS II & $40 \pm 5.7$ \\
SOFA score & $7 \pm 2.5$ \\
Total days of ECMO & $14(13-19)$ \\
Total day of MV & $19(17-33)$ \\
ICU length of stay (days) & $33(19-36)$ \\
Outcome (survivors) & $5 / 5$
\end{tabular}

SAPS II and SOFA score are calculated on ICU admission day, data are reported as mean \pm standard deviation or median (25-75\%)

$M V$ mechanical ventilation, $I C U$ intensive care unit nursing was performed in 23 cases out of 25 recorded nursing episodes. In two cases, occurred on two consecutive days in the same patient, daily nursing care was not performed due to active bleeding causing hemorrhagic shock. In the 23 remaining nursing episodes, no signs of active bleeding were recorded.

Globally daily nursing care lasted a median of 58 (46-65) min; sponge bath and elevation with scooping stretcher were the longer steps lasting a median of $14 \mathrm{~min}$ each (10-18, and 11-16, respectively), while change position of endotracheal tube and dressing change lasted 6

Table 2 Baseline conditions

\begin{tabular}{|c|c|}
\hline ICU day & $9(8-14)$ \\
\hline ECMO day & $8(7-11)$ \\
\hline SOFA score & $4(3-4)$ \\
\hline \multicolumn{2}{|l|}{ Sedation } \\
\hline Hypnotic agents & $\begin{array}{l}\text { Propofol } 182 \pm 68 \mathrm{mg} / \mathrm{h} \text { or } \\
\text { Midazolam } 7.9 \pm 1.8 \mathrm{mg} / \mathrm{h}\end{array}$ \\
\hline Opioids & $\begin{array}{l}\text { Fentanyl } 175 \pm 45 \mathrm{mcg} / \mathrm{h} \text { or } \\
\text { Sufentanyl } 0.32 \pm 0.09 \mathrm{mcg} / \\
\mathrm{kg} / \mathrm{min}\end{array}$ \\
\hline $\mathrm{BIS}^{\mathrm{a}}$ & $50 \pm 14$ \\
\hline $\mathrm{RSS}^{\mathrm{b}}$ & $5 \pm 1$ \\
\hline \multicolumn{2}{|l|}{ Mechanical ventilation } \\
\hline $\mathrm{FiO}_{2} \mathrm{NL}$ & $0.6 \pm 0.1$ \\
\hline TV $(\mathrm{ml} / \mathrm{kg})$ & $3.1(2.5-4.4)$ \\
\hline $\mathrm{RR}(\mathrm{bpm})$ & $10(10-16)$ \\
\hline PEEP $\left(\mathrm{cmH}_{2} \mathrm{O}\right)$ & $16 \pm 1.6$ \\
\hline $\begin{array}{l}\text { Number of nursing episodes in } \\
\text { paralyzed patients }\end{array}$ & 12 \\
\hline $\begin{array}{l}\text { Number of nursing episodes in } \\
\text { patients during assisted } \\
\text { ventilation }\end{array}$ & 11 \\
\hline \multicolumn{2}{|l|}{$\mathrm{ABG}$} \\
\hline $\mathrm{PaO}_{2} \mathrm{mmHg}$ & $77.9 \pm 19$ \\
\hline $\mathrm{PaCO}_{2} \mathrm{mmHg}$ & $52.9 \pm 5.9$ \\
\hline $\mathrm{pH}$ & $7.406 \pm 0.028$ \\
\hline $\mathrm{HCO}_{3-} \mathrm{mmol} / \mathrm{L}$ & $32.4 \pm 3$ \\
\hline Lactate $\mathrm{mmol} / \mathrm{L}$ & $1.5 \pm 0.7$ \\
\hline \multicolumn{2}{|l|}{ ECMO } \\
\hline $\mathrm{BF} \mathrm{L} / \mathrm{min}$ & $3.1 \pm 1$ \\
\hline RPM & $2532 \pm 464$ \\
\hline $\mathrm{GF} \mathrm{L} / \mathrm{min}$ & $4.4 \pm 1.6$ \\
\hline $\mathrm{FiO}_{2} \mathrm{ML}$ & $0.8 \pm 0.2$ \\
\hline
\end{tabular}

Data are reported as mean \pm standard deviation or median (25-75 \%). Level of sedation was evaluated with BIS in paralyzed patients, otherwise with RSS

$I C U$ day day of ICU since admission, ECMO day days of ECMO support, $\mathrm{FiO}_{2} \mathrm{NL}$ inspiratory oxygen fraction in natural lung, $A B G$ arterial blood gases, $\mathrm{GF}$ ECMO gas flow, $\mathrm{FiO}_{2} \mathrm{ML}$ inspiratory oxygen fraction in artificial (membrane) lung

${ }^{\text {a }}$ In paralyzed patients

b In patients during assisted mechanical ventilation 
Table 3 Adverse events

\begin{tabular}{|c|c|c|c|c|c|c|}
\hline Adverse events & $n$ & $\begin{array}{l}\text { Median no of adverse } \\
\text { events for episode }\end{array}$ & $\begin{array}{l}\text { Baseline value } \\
\text { before event }\end{array}$ & $\begin{array}{l}\text { Worse value reached } \\
\text { during each event }\end{array}$ & $\begin{array}{l}\text { Average duration } \\
\text { (min) }\end{array}$ & $p$ \\
\hline Hypertension $(\mathrm{mmHg})$ & 29 & $1(1-2)$ & $123 \pm 21[81-161]$ & $161 \pm 19[128-188]$ & $27 \pm 18$ & $<0.001$ \\
\hline Desaturation (\%) & 28 & $1(1-2)$ & 97 (95-99) [89-100] & 91 (84-94) [68-97] & $25 \pm 20$ & $<0.001$ \\
\hline $\mathrm{SvO}_{2}$ reduction $(\%)$ & 17 & $1(0-1)$ & $81(78-88)$ [74-92] & $74(68-84)$ [61-87] & $25 \pm 21$ & $<0.001$ \\
\hline Tachycardia (bpm) & 14 & $0(0-1)$ & $102 \pm 17[60-122]$ & $120 \pm 17[84-140]$ & $23 \pm 17$ & $<0.001$ \\
\hline $\mathrm{BF}$ reduction $(\mathrm{L} / \mathrm{min})$ & 10 & $0(0-1)$ & $2.4(2.1-3.3)[2-4.8]$ & $1.7(0.5-2.1)[0.4-3.7]$ & $14 \pm 11$ & 0.002 \\
\hline Tachypnea (bpm) & 25 & $3(2-3)$ & $17 \pm 5[8-26]$ & $28 \pm 5[19-35]$ & $9 \pm 3$ & $<0.001$ \\
\hline TV elevation $(\mathrm{ml} / \mathrm{kg})$ & 17 & $2(2-3)$ & $3.9 \pm 0.6[2.9-5.2]$ & $5.8 \pm 0.8[4.7-7.5]$ & $6 \pm 3$ & $<0.001$ \\
\hline MV elevation (L/min) & 25 & $3(2-3)$ & $6 \pm 1.9[3.1-10.2]$ & $9.7 \pm 2.5[5.5-15]$ & $8 \pm 4$ & $<0.001$ \\
\hline
\end{tabular}

Data are reported as mean $\pm \mathrm{SD}$ or as median $(25-75 \%)$ and as (min-max)

Baseline value mean value of each physiological parameter recorded over the $3 \mathrm{~min}$ before the beginning of the adverse event, desaturation reduction in $\mathrm{SpO}_{2}$ equal or greater than 3 points, hypertension elevation in systolic blood pressure greater than $15 \%$ from baseline, $\mathrm{SvO}$ reduction reduction in $\mathrm{SvO}_{2}$ greater than 3 points from baseline, tachycardia elevation in heart rate greater than $15 \%$ from baseline, $B F$ reduction reduction in $\mathrm{BF}$ greater than $15 \%$ from baseline, tachypnea elevation in respiratory rate greater than $30 \%$ from baseline, TV reduction reduction in TV greater than $25 \%$ from baseline, TV elevation elevation in TV greater than $25 \%$ from baseline, MV elevation elevation in MV greater than $25 \%$ from baseline, $n$ number of events, $\mathrm{SpO}_{2}$ arterial oxygen saturation, $\mathrm{SBP}$ systolic blood pressure, $\mathrm{Sv} \mathrm{O}_{2}$ mixed venous oxygen saturation, $H R$ heart rate, $B F$ ECMO blood flow

(5-8) and 12 (10-16) min, respectively. Total nursing time was longer than the sum of the length of each step because it includes also pauses in which, for example, nurses called physicians to administer boluses of sedation.

Sponge bath and elevation with scooping stretcher were performed in all the 23 cases, while change position of endotracheal tube and dressing change were performed, respectively, in 16 and 20 cases, since deemed unnecessary as performed for other reasons during previous nursing shifts.

All the nursing episodes started with sponge bath except in one case and were usually followed by elevation with scooping stretcher $(16 / 23,69 \%)$.

In all the nursing episodes, we recorded at least two adverse events, with a median value of 4 (3-5) adverse events for each nursing episode (respiratory adverse events excluded). Frequency, magnitude and distribution of each adverse event are reported in Table 3 and Fig. 1.

During nursing, we observed a significant reduction of $\mathrm{BF}(-29 \%), \mathrm{SvO}_{2}(-8.6 \%)$ and $\mathrm{SpO}_{2}(-6.2 \%)$ and a significant elevation of $\mathrm{HR}(+18 \%)$ and SBP $(+31 \%)$. Arterial oxygen desaturation and systemic blood hypertension were the most common recorded adverse events. In assisted mechanical ventilation patients, we also analyzed modification in respiratory pattern during nursing care (11 nursing episodes). Ventilator parameters were not recorded in 2 episodes due to technical problems. We observed 25 significant elevation in minute ventilation $(+62 \%)$ : in 17 cases, we recorded an increase of both respiratory rate and tidal volume, while in 4 times the elevation in minute ventilation was due to an elevation in respiratory rate with a constant tidal volume, and finally in 1 case (with four events of elevation in minute ventilation) tidal volume was not recorded due to technical problems. Considering respiratory adverse events, we observed a total amount of 123 adverse events, 70 in spontaneous breathing patients while 53 in patients in controlled mechanical ventilation; however, no statistical difference was observed between the two groups ( $p=0.064)$.

The nursing step with the higher rate of adverse events was the elevation with scooping stretcher. Sponge bath, usually performed at the beginning of nursing, was instead associated with the higher rate of hypertensive events (see Table 4). No adverse events were recorded during dressing change. Elevation with scooping stretcher often caused a significant reduction of $\mathrm{BF}$ which leaded to reduction in $\mathrm{SvO}_{2}$ and arterial desaturation. The elevation in minute ventilation was observed mainly during sponge bath and elevation with scooping stretcher (see Table 4).

\section{Sedation management}

Fourteen boluses of sedatives were administrated before nursing. Seventeen boluses of sedatives were administrated during nursing by the clinicians if deemed necessary (see Table 4). In 8 daily nursing episodes, boluses of sedation were administered before and during nursing care. In 6 nursing episodes, additional boluses of sedation were administered only before and in 4 nursing episodes only during nursing care. Finally in 5 nursing episodes, patients did not receive any additional bolus of sedation (see Electronic Supplementary Material, Table 1S). No statistically significant differences in observed adverse event were found in the different categories of sedation. 


\section{Desaturation}

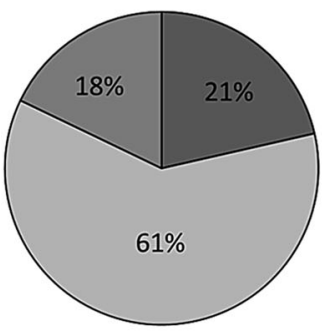

Hypertension

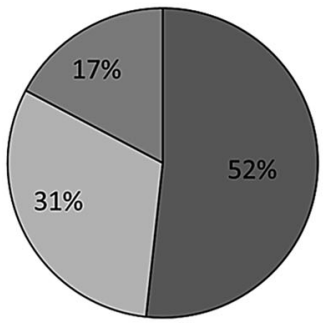

$\mathrm{SvO}_{2}$ reduction

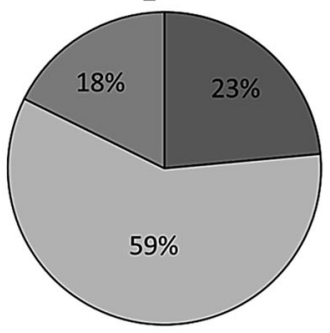

$\square$ sponge bath

$\square$ elevation with scooping stretcher

$\square$ change ETT

Fig. 1 Frequency of adverse events in each nursing phase. No adverse event was recorded during "dressing change" phase

\section{Reduction in $\mathrm{BF}$, desaturation and reduction in $\mathrm{SvO}_{2}$}

Reduction in $\mathrm{BF}$ led to desaturation and reduction in $\mathrm{SvO}_{2}$ in $50 \%$ of cases, while in 3 cases drop in BF was associated only with desaturation and in one case only with reduction in $\mathrm{SvO}_{2}$. Finally, in one case, reduction in BF was isolated (see Electronic Supplementary Material, Table $1 \mathrm{~S}$ ). We found an inverse correlation between baseline $\mathrm{BF}$ and the magnitude of event of desaturation and reduction in $\mathrm{SvO}_{2}$. All the events of desaturation greater than 14 points of reduction in $\mathrm{SpO}_{2}$ were observed in patients with a baseline $\mathrm{BF}$ lower or equal to $3 \mathrm{~L} / \mathrm{min}$; similarly, all the events of reduction in $\mathrm{SvO}_{2}$ greater than 8 points were observed in patients with a baseline BF lower or equal to $3 \mathrm{~L} / \mathrm{min}$.

\section{Discussion}

Daily nursing was performed in $92 \%$ of cases $(23 / 25)$, with a minimum of two adverse events per episode and was associated with hypertension and tachycardia, mostly at the beginning of nursing, while desaturation, reduction in $\mathrm{SvO}_{2}$ and in $\mathrm{BF}$ were common events during elevation with scooping stretcher. In spontaneous breathing patients, elevation in minute ventilation was frequent. Additional bolus of sedation was required before and/or during nursing in most of the nursing episodes. The reduction in BF was usually associated with desaturation and/or reduction in $\mathrm{SvO}_{2}$. Finally with lower baseline blood flow, we observed the higher magnitude of desaturation and reduction in $\mathrm{SvO}_{2}$.

Daily nursing is an essential activity in patient care, it improves patient comfort, may reduce the incidence of iatrogenic infections, and it is critical to assess skin integrity, especially in hidden areas such as the back. Daily nursing in critical patients undergoing ECMO is also important to evaluate bleeding and vascular access, but due to the extremely critical conditions of these patients, it is more difficult and requires trained personnel. Indeed, in our case series, several adverse events, requiring the intervention of a physician, were recorded during each daily nursing.
Table 4 Number of adverse events occurred in each nursing phase

\begin{tabular}{llll}
\hline & Sponge bath & Elevation with scooping stretcher & Change position of ETT \\
\hline$\uparrow \mathrm{SBP}$ & 15 & 9 & 5 \\
$\uparrow \mathrm{HR}$ & 6 & 5 & 3 \\
$\downarrow \mathrm{MV}$ & 10 & 11 & 4 \\
$\downarrow \mathrm{SpO}_{2}$ & 4 & 19 & 5 \\
$\downarrow \mathrm{SvO}_{2}$ & 3 & 11 & 3 \\
$\downarrow \mathrm{BF}$ & 2 & 8 & 0 \\
Sedation $^{\text {a }}$ & 6 & 5 & 6
\end{tabular}

$\uparrow \mathrm{SBP}$ hypertensive event, $\downarrow \mathrm{SvO}_{2}$ reduction in $\mathrm{SvO}_{2}, \downarrow \mathrm{SpO}_{2}$ desaturation event, $\uparrow H R$ tachycardic event, $\downarrow$ $B F$ reduction in $\mathrm{BF}, \downarrow M V$ elevation in $\mathrm{MV}$

${ }^{a}$ Administration of bolus of sedation 
Sponge bath, the first step usually performed, was mostly associated with hypertensive and tachycardic events, probably to be referred to an inadequate level of sedation. Despite the use of warm water during the sponge bath, the average increase in systolic pressure was conspicuous, $31 \%$ of baseline, which lasted for slightly less than half an hour. Indeed, in spite of a good sedation plan before nursing, additional bolus was often required; thus, the sedation status should be carefully evaluated before and during nursing care and deepened if necessary. In vvECMO patients, an inadequate sedation plan may lead also to increase in oxygen consumption, elevation in cardiac output (CO) and subsequent arterial desaturation. In spontaneous breathing patients, mobilization and painful stimulation may increase work of breathing leading to a critical elevation in minute ventilation.

Veno-venous ECMO improves arterial oxygenation through elevation in mixed venous saturation; thus, BF level and $\mathrm{SvO}_{2}$ and $\mathrm{SpO}_{2}$ are strictly dependent. Since our patients were connected to centrifugal pumps set to deliver a constant number of rpm, the BF could change during the nursing care, specifically a reduction in $\mathrm{BF}$ was often recorded during elevation with the scooping stretcher (average $\mathrm{BF}$ reduction $29 \%$ ). The magnitude of these changes may depend on the volume status of the patients, indeed a normovolemic or relative hypervolemic patient could better tolerate this maneuver. Higher blood flow (i.e., 4-5 L/ min) usually required an higher volume status to ensure an adequate venous drainage without hemolysis; thus, patients with higher blood flow had fewer episodes of drop in BF and, consequently, fewer desaturation and reduction in $\mathrm{SvO}_{2}$. During the drop in BF, a slight reduction in RPM could be tested as a technique to stabilize the BF.

According to the results of this study, our nursing care protocol was modified, specifically, to avoid reduction in $\mathrm{BF}$, hence elevation with scooping stretcher was eliminated and sheets replacement and back hygiene are now performed only with gentle mobilization of the patient on the side, which seems to reduce the alterations in BF.

This study had several limitation: we analyzed a small group of patient, we performed an observational study without any control group, and we did not standardize the ECMO day in which we performed the study, indeed patients could be in respiratory weaning or in the early acute phase of ARDS.

\section{Conclusion}

Nursing care may have a significant impact on physiologic parameters of patients undergoing vv-ECMO support. Tachycardia, hypertension and reduction in oxygenation were commonly recorded and sedation level should be carefully evaluated before and/or during nursing. Furthermore, in spontaneous breathing patients minute ventilation may significantly increase. We suggest that daily nursing care should be performed only when medical personnel are present or readily available to adjust sedation plan, ventilator or ECMO setting and to treat critical alteration in vital signs.

\section{Compliance with ethical standards}

Conflict of interest We acknowledge the following potential conflicts of interest. Dr. Pesenti received payment for lectures and service on speaker bureau from Maquet and Novalung. The remaining authors have disclosed that they do not have any potential conflicts of interest.

Source of funding Funding for this study was provided by the inhouse research funds from Dipartimento Scienze della Salute, Università di Milano-Bicocca, Milano, Italy.

\section{References}

1. Elliott D, Aitken L, Chaboyer W. Critical care nursing. Sydney: Mosby Elsevier; 2007.

2. Coyer FM, O'Sullivan J, Cadman N. The provision of patient personal hygiene in the intensive care unit: a descriptive exploratory study of bed-bathing practice. Aust Crit Care. 2011;24:198-209.

3. Atkins PJ, Hapshe E, Riegel B. Effects of a bed-bath on mixed venous oxygen saturation and heart rate in coronary artery bypass graft patients. Am J Crit Care. 1994;3:107-15.

4. Copel LC, Stolarik A. Impact of nursing care activities on $\mathrm{SvO}_{2}$ levels of postoperative cardiac surgery patients. Cardiovasc Nurs. 1991;27:1-5.

5. Doering LV. The effect of positioning on hemodynamics and gas exchange in the critically ill: a review. Am J Crit Care. 1993;2:208-16.

6. Johnston BL, Watt EW, Fletcher GF. Oxygen consumption and hemodynamic and electrocardiographic responses to bathing in recent post-myocardial infarction patients. Heart Lung. 1981;10:666-71.

7. Lewis P, Nichols E, Mackey G, et al. The effect of turning and backrub on mixed venous oxygen saturation in critically ill patients. Am J Crit Care. 1997;6:132-40.

8. Noll ML, Duncan CA, Fountain RL, Weaver L, Osmanski VP, Halfman S. The effect of activities on mixed venous oxygen saturation $\left(\mathrm{SvO}_{2}\right)$ in critically ill patients. Heart Lung. 1991;20:301.

9. Shively M. Effect of position change on mixed venous oxygen saturation in coronary artery bypass surgery patients. Heart Lung. 1988;17:51-9.

10. Tidwell SL, Ryan WJ, Osguthorpe SG, Paull DL, Smith TL. Effects of position changes on mixed venous oxygen saturation in patients after coronary revascularization. Heart Lung. 1990;19:574-8.

11. Winslow EH, Clark AP, White KM, Tyler DO. Effects of a lateral turn on mixed venous oxygen saturation and heart rate in critically ill adults. Heart Lung. 1990;19:557-61.

12. Happ MB, Tate AJ, Swigart VA, DiVirgilio-Thomas D, Hoffman LA, et al. Wash and wean: bathing patients undergoing weaning trials during prolonged mechanical ventilation. Heart Lung. 2010;39:S47-56. 
13. Patroniti N, Zangrillo A, Pappalardo F, Peris A, Cianchi G, Braschi A, et al. The Italian ECMO network experience during the 2009 influenza $\mathrm{A}(\mathrm{H} 1 \mathrm{~N} 1)$ pandemic: preparation for severe respiratory emergency outbreaks. Intensive Care Med. 2011;37:1447-57.

14. Mauri T, Foti G, Zanella A, Bombino M, Confalonieri A, Patroniti $\mathrm{N}$, et al. Long-term extracorporeal membrane oxygenation with minimal ventilatory support: a new paradigm for severe ARDS? Minerva Anestesiol. 2012;78:385-9.

15. Grasselli G, Foti G, Patroniti N, Giuffrida A, Cortinovis B, Zanella A, et al. A case of ARDS associated with influenza AH1N1 infection treated with extracorporeal respiratory support. Minerva Anestesiol. 2009;75:741-5. 